Zürich University Preprint

ZU-TH 4/96

\title{
Asymptotic Behavior of the Einstein-Yang-Mills-Dilaton System for a Closed Friedmann-Lemaitre Universe"
}

\author{
By Reto Eggenschwiler, David Scialom and Norbert Straumann \\ Institute of Theoretical Physics, University of Zürich, Winterthurerstrasse 190, \\ CH-8057 Zürich, Switzerland
}

\begin{abstract}
We study the coupled Einstein-Yang-Mills-Dilaton (EYMD) equations for a FriedmannLemaitre universe with constant curvature $k=1$. Our detailed analysis is restricted to the case where the dilaton potential and the cosmological constant vanish. Also assuming a static gauge field, we present analytical and numerical results on the behavior of solutions of the EYMD equations. For different values of the dilaton coupling constant we analyze the phase portrait for the time evolution of the dilaton field and give the behavior of the scale factor. It turns out that there are no inflationary stages in this model.
\end{abstract}

\section{Introduction}

For a long time the matter used in most cosmological models was described in terms of ideal or viscous hydrodynamics. This is certainly adequate at least back to the time of nucleosynthesis, because - to a first approximation - we are then allowed to regard the matter as a mixture of pressure-free matter (baryons, WIMPS, etc...) and radiation (photons, neutrinos). At very high energies the fluid description has to be replaced by field theoretical models (and, when approaching the Planck scale, by yet unknown theories of matter).

\footnotetext{
*This work was supported by the Swiss National Science Foundation.
} 
With the success of gauge field theories, and especially in connection with various proposed inflationary scenarios [1], field theoretical models became very popular in cosmology. A description of topological defects, which could be created in cosmological phase transition, requires also field theoretical models which allow for spontaneous symmetry breaking.

Inflationary stages appear more or less naturally in many models, but most of those which lead to successful inflation are very phenomenological in nature and are not based directly on "fundamental" field theories. Since inflation is a very attractive general idea and for other reasons as well, it is important to study the evolution of a large variety of cosmological models based on interesting field theoretical matter descriptions. In addition to Yang-Mills fields, the existence of a dilaton field is theoretically well motivated on the basis of superstring theory [2] and Kaluza-Klein theories [3].

In the present work we study the coupled Einstein-Yang-Mills-Dilaton (EYMD) equations for a Friedmann-Lemaitre (Robertson-Walker) space-time. The basic equations for this model lead to a dynamical system of six first-order nonlinear ordinary differential equations, which we discuss with the modern techniques of the theory of dynamical systems. In view of the rather high dimension of phase space this goal is not easy to achieve. In a first step we therefore analyze in detail only a restricted class of solutions. We do so by setting the cosmological constant and the dilaton potential equal to zero, and also require that the YangMills field is static, which can be done consistently. In this case the phase space becomes three-dimensional. We hope that our analysis of this reduced system is at least methodically of some interest. An extension of the discussion to the full system is planned.

In a first step we compactify the phase space such that the dynamical system can be extended to the enlarged space and determine the critical points. With one exception, all critical points are either located at infinity of the original phase space or in the unphysical region. We analyze the nature of these singular points and the qualitative behavior of the flow in their vicinity. In general the equilibrium points are hyperbolic. However, for special values of the dilaton coupling constant there exists a critical point in the asymptotic physical region which becomes non-hyperbolic. For this case we use the theory of normal forms and apply the Poincaré-Dulac theorem to get rid of the nonresonant nonlinear terms up to third order. Analytical formula which are valid near the critical points are given in all cases.

The asymptotic behavior provides one with the initial conditions for a numerical study of the phase portrait. Special attention is given to the value of the dilaton coupling constant which is obtained from superstring theory. Unfortunately, and not unexpectedly, we do not find any inflationary stages.

The paper is organized as follows. In section 2 we derive the basic equations. Their analysis for the above-mentioned assumptions is performed in section 3. We analytically determine the asymptotic behavior of the solutions near the singular points. Section 4 is devoted to numerical results. The behavior of the scale factor as well as the phase portrait of the dilaton field are given. A short summary concludes the paper. 


\section{Basic Equations}

The metric of the Friedmann-Lemaitre universe with constant curvature $k=1$ is given by

$$
g=d t^{\prime 2}-a^{2}\left(t^{\prime}\right) h=a^{2}(t)\left[d t^{2}-h\right]
$$

where $h$ is the standard metric on $S^{3}$ and $t$ is the conformal time related to cosmic (proper) time by $d t^{\prime}=a(t) d t$.

We work in the "Einstein conformal frame" in which the action for the EYMD system is given by

$$
S=\frac{1}{4 \pi} \int\left(-\frac{R}{4 G}-\frac{\Lambda}{2 G}+\frac{1}{2}(\partial \phi)^{2}-V(\phi)-\frac{e^{-2 \kappa \phi}}{4} F^{2}\right) \eta,
$$

where $F=d A+A \wedge A, \kappa$ denotes the dilatonic coupling constant and $\eta$ is the volume form. $A$ and $\phi=\phi(t)$ are the $S U(2)$ Yang-Mills potential and the dilaton field, respectively. Both are homogeneous and isotropic.

Since the group manifold of $S U(2)$ is $S^{3}$, there is a natural ansatz for a homogeneous isotropic $S U(2)$ gauge field involving a single function of time [6]:

$$
A=f(t) \Theta
$$

where $\Theta$ is the Maurer-Cartan form on $S^{3}$ regarded as $S U(2)$. Note that $t$ and $f$ must be considered dimensionless while the scale factor $a$ has the dimension of a length. The canonical metric $h$ on $S^{3}$ can also be expressed in terms of $\Theta$ : If we decompose the $s u(2)$-valued form according to

$$
\Theta=\sum \theta^{j} \tau_{j}
$$

where $\tau_{j}=-i \sigma_{j}\left(\sigma_{j}\right.$ are the Pauli matrices $)$, then the $\theta^{j}$ are left invariant 1 -forms and

$$
h=\delta_{i j} \theta^{i} \otimes \theta^{j}
$$

A simple way to verify the normalization is to use the following parameterization of $S U(2)$ in terms of $x=\left(x^{1}, x^{2}, x^{3}, x^{4}\right) \in S^{3}$ :

$$
U=x^{4}-x^{j} \tau_{j}=\left(\begin{array}{cc}
x^{4}+i x^{3} & x^{2}+i x^{1} \\
-x^{2}+i x^{1} & x^{4}-i x^{3}
\end{array}\right) .
$$

The Maurer-Cartan form is then $U^{-1} d U$ and the $\theta^{i}$ are given by

$$
\theta^{i}=-\frac{1}{2} \operatorname{tr}\left[\tau_{i} U^{-1} d U\right] \quad(i=1,2,3)
$$

We define an orthonormal basis of one-forms by

$$
\hat{\theta}^{0}:=a d t, \hat{\theta}^{i}:=a \theta^{i} \quad(i=1,2,3) .
$$

The metric (2.1) then reads

$$
g=\hat{g}_{\mu \nu} \hat{\theta}^{\mu} \otimes \hat{\theta}^{\nu}, \quad \hat{g}_{\mu \nu}=\operatorname{diag}(1,-1,-1,-1) .
$$


Variation of the action with respect to the metric yields the Einstein field equations

$$
\hat{G}_{\mu \nu}=8 \pi G \hat{T}_{\mu \nu}+\Lambda \hat{g}_{\mu \nu},
$$

with

$$
\begin{aligned}
& \hat{T}_{00}=\frac{1}{4 \pi}\left\{\frac{\dot{\phi}^{2}}{2 a^{2}}+V(\phi)+\frac{3}{2 a^{4}}\left[\dot{f}^{2}+4\left(f^{2}-f\right)^{2}\right] e^{-2 \kappa \phi}\right\}, \\
& \hat{T}_{i j}=\frac{1}{4 \pi}\left\{\frac{\dot{\phi}^{2}}{2 a^{2}}-V(\phi)+\frac{1}{2 a^{4}}\left[\dot{f}^{2}+4\left(f^{2}-f\right)^{2}\right] e^{-2 \kappa \phi}\right\} \delta_{i j},
\end{aligned}
$$

and $\hat{T}_{0 i}=0$. The (00) component of Einstein's field equations is the constraint equation

$$
H^{2}+1=G\left\{\frac{1}{3} \dot{\phi}^{2}+\frac{2 a^{2}}{3} V(\phi)+\frac{1}{a^{2}}\left[\dot{f}^{2}+4\left(f^{2}-f\right)^{2}\right] e^{-2 \kappa \phi}\right\}+\frac{\Lambda}{3} a^{2},
$$

with the Hubble parameter $H=\dot{a} / a$, where an overdot means the derivative with respect to the conformal time $t$. For the spatial components we have

$$
-\left[2 \dot{H}+H^{2}+1\right]=G\left\{\dot{\phi}^{2}-2 a^{2} V(\phi)+\frac{1}{a^{2}}\left[\dot{f}^{2}+4\left(f^{2}-f\right)^{2}\right] e^{-2 \kappa \phi}\right\}-\Lambda a^{2} .
$$

The dilaton and the YM equation are obtained from variations of the action with respect to $\phi$ and $f$, repectively,

$$
\begin{aligned}
\ddot{\phi}+2 H \dot{\phi}+a^{2} \frac{\partial V}{\partial \phi}+\frac{3 \kappa}{a^{2}}\left[\dot{f}^{2}-4\left(f^{2}-f\right)^{2}\right] e^{-2 \kappa \phi} & =0, \\
\ddot{f}-2 \kappa \dot{\phi} \dot{f}+4 f(2 f-1)(f-1) & =0 .
\end{aligned}
$$

The system is fully determined by the independent equations (2.11), (2.13) and (2.14). In fact, one can (easily) show that Eq. (2.12) is a consequence of the others. This is a reflection of the Bianchi identities.

From Eq. (2.14) we see that the static gauge field solutions are given by $f=0,1 / 2,1$. Only the case $f=1 / 2$ is of interest, because $f=0$ corresponds to a vanishing gauge field $(A=0)$ and $f=1$ is a pure gauge $(A=\Theta)$.

A systematic study of the complete system is rather involved. We confine ourselves to the special case of a static gauge field $f=1 / 2$ and choose in addition $V=0$ and $\Lambda=0$. The set of differential equations (2.11)-(2.14) then reduces to a three-dimensional dynamical system. In order to analyze this reduced system, we use the same procedure as in [7]. We determine the singular points [8] (see page 15), including those lying at infinity of the phase space, and then find analytically the asymptotic behavior for the solutions near these points.

\section{Asymptotic Behavior}

Setting $f=1 / 2, V=0, \Lambda=0$ and introducing a "proper" time coordinate $\tilde{t}$ according to

$$
d \tilde{t}=\frac{d t}{a(t)}
$$


we obtain the transformed system of basic equations

$$
\begin{aligned}
\phi_{\tilde{t} \tilde{t}} & =-\tilde{H} \phi_{\tilde{t}}+\frac{3 \kappa}{4} e^{-2 \kappa \phi}, \\
\tilde{H}_{\tilde{t}} & =\tilde{H}^{2}-G\left[\frac{2}{3} \phi_{\tilde{t}}^{2}+\frac{1}{4} e^{-2 \kappa \phi}\right], \\
\tilde{H}^{2} & +a^{2}=G\left[\frac{1}{3} \phi_{\tilde{t}}^{2}+\frac{1}{4} e^{-2 \kappa \phi}\right],
\end{aligned}
$$

where $\tilde{H}=a_{\tilde{t}} / a$ and the subscript $\tilde{t}$ denotes the derivative with respect to this variable.

Equations (3.2) and (3.3) form a three-dimensional dynamical system in the phase space $\phi, \phi_{\tilde{t}}, \tilde{H}$. We now introduce the following dimensionless variables:

$$
\begin{aligned}
\tilde{t} \rightarrow \eta & =\sqrt{G} \tilde{t}, \\
\kappa \rightarrow \tilde{\kappa} & =\sqrt{\frac{3}{G}} \kappa, \\
\phi \rightarrow x & =\sqrt{\frac{G}{3}} \phi, \\
\phi_{\tilde{t}} \rightarrow y & =\frac{1}{\sqrt{3}} \phi_{\tilde{t}} \\
\tilde{H} \rightarrow z & =\frac{\tilde{H}}{\sqrt{G}}, \\
a \rightarrow \tilde{a} & =\frac{a}{\sqrt{G}} .
\end{aligned}
$$

Then Eqs. (3.2) and (3.3) become

$$
\begin{aligned}
& x_{\eta}=y, \\
& y_{\eta}=-y z+\frac{\tilde{\kappa}}{4} e^{-2 \tilde{\kappa} x}, \\
& z_{\eta}=z^{2}-2 y^{2}-\frac{1}{4} e^{-2 \tilde{\kappa} x},
\end{aligned}
$$

and the constraint (3.4) takes the form $y^{2}+\frac{1}{4} e^{-2 \tilde{\kappa} x}-z^{2}=\tilde{a}^{2}$ with $z=\tilde{a}_{\eta} / \tilde{a}$.

For the special case $\tilde{\kappa}=0$, these equations are equivalent to a one-dimensional mechanical system for $a(\eta)$ with the Lagrange function $L=T-V=\tilde{a}_{\eta}^{2}-\left(\tilde{a}^{4}-\tilde{a}^{2} / 4\right)$. Indeed, Eq. (3.6) immediately implies the relation $y=k / \tilde{a}$, where $k$ is an arbitrary constant. From the constraint equation we obtain the corresponding conserved "energy" $E=T+V=\tilde{a}_{\eta}^{2}+$ $\left(\tilde{a}^{4}-\tilde{a}^{2} / 4\right)=k^{2} \geq 0$. The solution with respect to the conformal time is

$$
\begin{aligned}
& \tilde{a}(t)=\frac{\sqrt{2}}{4}\left[1+\sqrt{1+64 k^{2}} \sin (2 t-\lambda)\right]^{\frac{1}{2}}, \\
& z(t)=\frac{\sqrt{2}}{4} \frac{\sqrt{1+64 k^{2}} \cos (2 t-\lambda)}{\left(1+\sqrt{1+64 k^{2}} \sin (2 t-\lambda)\right)^{\frac{1}{2}}}, \\
& x(t)=\frac{1}{2} \ln \left[\frac{\tan (t-\lambda / 2)+\sqrt{1+64 k^{2}}-8 k}{\tan (t-\lambda / 2)+\sqrt{1+64 k^{2}}+8 k}\right]+\text { const. } \quad t \in(0, \pi / 2+\lambda),
\end{aligned}
$$


where $\lambda$ is defined by

$$
\lambda:=\arcsin \left(\frac{1}{\sqrt{1+64 k^{2}}}\right) .
$$

If $\tilde{\kappa} \neq 0$, we use instead of $x$ the function

$$
u=\frac{e^{-\tilde{\kappa} x}}{2}>0 .
$$

Our system then takes the final form

$$
\begin{aligned}
& u_{\eta}=-\tilde{\kappa} u y, \\
& y_{\eta}=-y z+\tilde{\kappa} u^{2}, \\
& z_{\eta}=z^{2}-u^{2}-2 y^{2},
\end{aligned}
$$

with the constraint

$$
u^{2}+y^{2}-z^{2}=\tilde{a}^{2} .
$$

where we recall that $z$ is the Hubble parameter with respect to $\eta$,

$$
z=\frac{\tilde{a}_{\eta}}{\tilde{a}} .
$$

The system of equations (3.9)-(3.11) possesses the following symmetries:
a) $u \rightarrow-u$
b) $\eta \rightarrow-\eta$
$y \rightarrow-y$
$z \rightarrow-z$
c) $\tilde{a} \rightarrow-\tilde{a}$
d) $y \rightarrow-y$

According to a) the plane $u=0$ is a two-dimensional invariant subspace of the phase space; it separates solutions with $u>0$ from those with $u<0$. Trajectories can reach this plane asymptotically, but they never penetrate it. We are not interested in solutions with $u \equiv 0$; for these the change of variables given by Eq. (3.8) would no longer be valid.

Equations (3.8) and (3.10) imply that the trajectories of our model lie in the region of phase space exterior to the cone $u^{2}+y^{2}-z^{2}=0$ where $u>0$ (see Fig. 1 ).

We also note that the sections of the trajectories lying in the upper half of the phase space $(z>0)$ correspond to expansion $(H>0)$ and those in the lower $(z<0)$ to contraction $(H<0)$. When a trajectory intersects the plane $z=0(H=0)$, the scale factor $\tilde{a}$ attains its maximum, because $z_{\eta}=-\left(u^{2}+2 y^{2}\right)$ is strictly negative.

\subsection{The Singular Point at the Origin}

Looking for equilibrium states of the dynamical system (3.9) we immediately see that the origin $(u, y, z)=(0,0,0)$ is the only singular point not lying at infinity. However, this 


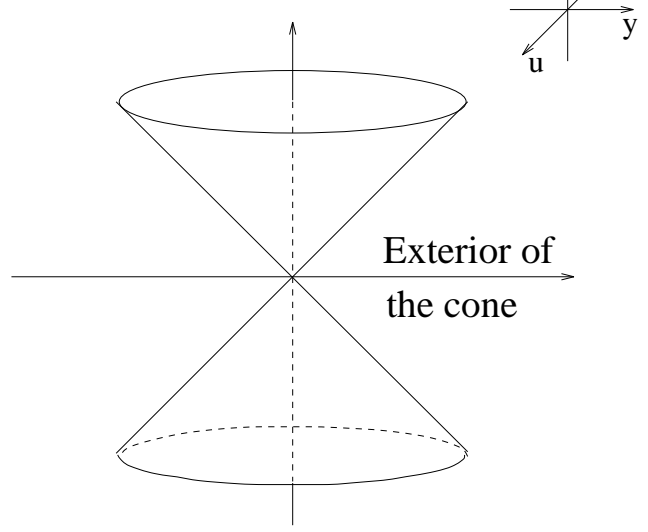

Figure 1: Physical domain.

critical point is not of much interest, because there are no trajectories originating from it which extend to the exterior of the cone nor does any trajectory (coming from the exterior of the cone) ever reach the origin: Suppose there would be a trajectory emerging from the origin $(u, y, z)=(0,0,0)$. Along this, $z=\tilde{a}_{\eta} / \tilde{a}$ has to be negative, since $z_{\eta}=-\left(y^{2}+\tilde{a}^{2}\right)$ is strictly negative. It follows that if $\tilde{a}_{\eta}>0\left(\tilde{a}_{\eta}<0\right)$ along the trajectory, then $\tilde{a}$ must be negative (positive). This is a contradiction because $\tilde{a}$ vanishes at the origin. With similar arguments one can prove that no trajectory can reach the origin from the exterior of the cone.

The repelling behavior of the singular point $(u, y, z)=(0,0,0)$ is nicely illustrated by considering the case where $\tilde{\kappa}=1$. Then the plane $z=-y$ is an invariant subspace and the phase portrait of the trajectories lying on this plane is given in Fig. 2. Furthermore, we can give the explicit solution. On the invariant plane $z=-y$ our system of Eqs. (3.9) reduces to

$$
\begin{aligned}
& u_{\eta}=u z, \\
& z_{\eta}=-z^{2}-u^{2},
\end{aligned}
$$

and the constraint equation becomes $u^{2}=\tilde{a}^{2}$. Eq. (3.13) is equivalent to the equation of motion of a one-dimensional mechanical system with the Lagrange function $L=T-V=$ $\tilde{a}_{\eta}^{2} / 2-\tilde{a}^{4} / 4$. The corresponding conserved "energy" is $E=T+V=\tilde{a}_{\eta}^{2} / 2+\tilde{a}^{4} / 4 \geq 0$. The solution with respect to the conformal time is

$$
\begin{aligned}
& \tilde{a}(t)=u(t)=(2 \sqrt{E} \sin (\sqrt{2} t))^{\frac{1}{2}}, \\
& z(t)=-y(t)=\frac{\sqrt{E} \cos \sqrt{2} t}{(\sqrt{E} \sin (\sqrt{2} t))^{\frac{1}{2}}} \\
& x(t)=-\frac{1}{2} \ln [8 \sqrt{E} \sin (\sqrt{2} t)] \quad t \in(0, \pi / \sqrt{2}) .
\end{aligned}
$$

In the limit $t \rightarrow 0$, the scale factor $\tilde{a}$ vanishes and the equation of state tends to $\epsilon=p$ (stiff matter), where $\epsilon=\hat{T}_{00}$ and $p=\frac{1}{3}\left(\hat{T}_{11}+\hat{T}_{22}+\hat{T}_{33}\right)$. At $t=\sqrt{2} \frac{\pi}{2}$, $\tilde{a}$ attains its maximum and 
the equation of state becomes $\epsilon=3 p$ which corresponds, for ordinary matter, to a extremely relativistic gas.

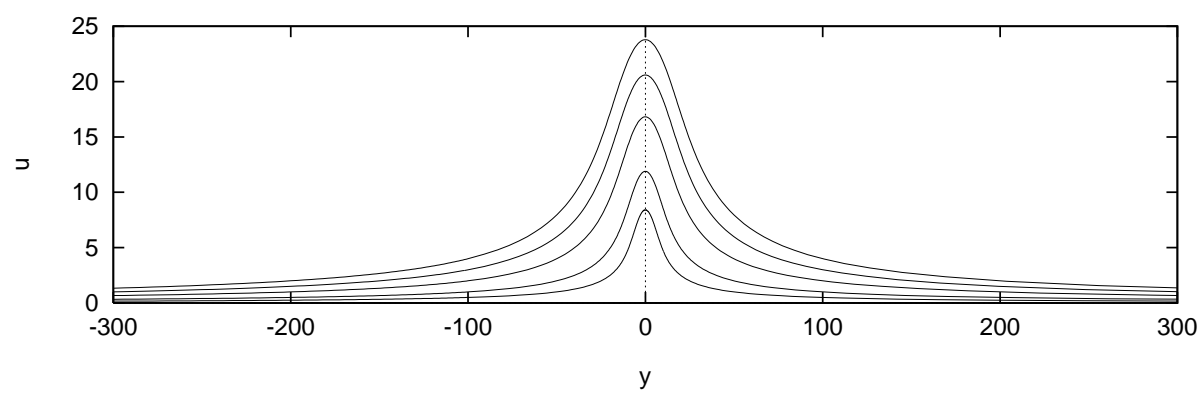

Figure 2: Phase portrait of the invariant plane $z=-y$.

\subsection{The Singular Points at Infinity}

In order to find and investigate the singular points at infinity it is convenient to compactify the phase space and complete it by the infinitely distant boundary $u^{2}+y^{2}+z^{2}=\infty$. We do so by going over from the Cartesian coordinates $u, y, z$ to the spherical coordinates

$$
u=r \cos \varphi \sin \vartheta, \quad y=r \sin \varphi \sin \vartheta, \quad z=r \cos \vartheta
$$

and by performing a subsequent transformation of the radius according to

$$
r=\frac{\rho}{1-\rho} \quad(0 \leq \rho<1)
$$

Also introducing a new time $\tau$ defined by

$$
d \eta=(1-\rho) d \tau
$$

and using the variables $\rho, \vartheta, \varphi, \tau$, our system (3.9) assumes the form

$$
\begin{aligned}
\rho_{\tau} & =\rho^{2}(1-\rho) \cos \vartheta\left[\cos (2 \vartheta)-2 \sin ^{2} \vartheta \sin ^{2} \varphi\right], \\
\vartheta_{\tau} & =-\rho \sin \vartheta \cos (2 \vartheta)\left[1+\sin ^{2} \varphi\right], \\
\varphi_{\tau} & =\rho \cos \varphi[\tilde{\kappa} \sin \vartheta-\sin \varphi \cos \vartheta] .
\end{aligned}
$$

For $0 \leq \rho<1$, Eqs. (3.9) are equivalent to the system (3.17), but the latter admits a smooth continuation to the boundary $\rho=1$. Thus, in the variables $0 \leq \rho \leq 1,0 \leq \vartheta \leq \pi$, $0 \leq \varphi \leq 2 \pi$, the phase space of the system (3.17) is compact. Each point of the original phase space is mapped along its radius vector to a certain point inside the unit ball, while the points $u^{2}+y^{2}+z^{2}=\infty$ at infinity are mapped to its surface $\rho=1$. From Eqs. (3.17) it is readily seen that on this surface the system has several singular points, their number and nature depending on the value of $\tilde{\kappa}$. Due to the symmetry transformation d) it is sufficient 


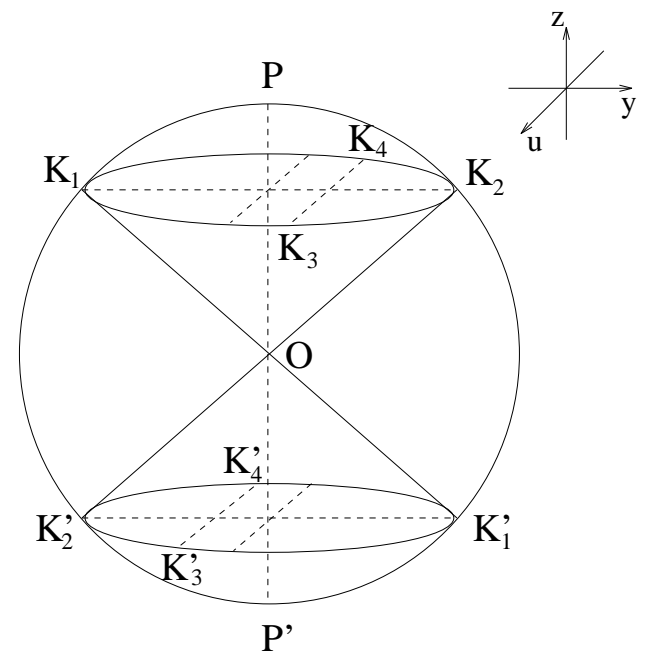

Figure 3: Singular points at infinity.

to consider only positive values of $\tilde{\kappa}$ (i.e. $\tilde{\kappa}>0$ ). There are at least six singular points which will be denoted by (see Fig. 3)

$$
\begin{array}{lll}
P(\vartheta=0), & K_{1}\left(\left(\vartheta_{1}, \varphi_{1}\right)=(\pi / 4,3 \pi / 2)\right), & K_{2}\left(\left(\vartheta_{2}, \varphi_{2}\right)=(\pi / 4, \pi / 2)\right), \\
P^{\prime}\left(\vartheta^{\prime}=\pi\right), & K_{1}^{\prime}\left(\left(\vartheta_{1}^{\prime}, \varphi_{1}^{\prime}\right)=(3 \pi / 4, \pi / 2)\right), & K_{2}^{\prime}\left(\left(\vartheta_{2}^{\prime}, \varphi_{2}^{\prime}\right)=(3 \pi / 4,3 \pi / 2)\right) .
\end{array}
$$

For $\tilde{\kappa}<1$ we have the four additional points

$$
\begin{array}{ll}
K_{3}\left(\left(\vartheta_{3}, \varphi_{3}\right)=(\pi / 4, \arcsin \tilde{\kappa})\right), & K_{4}\left(\left(\vartheta_{4}, \varphi_{4}\right)=(\pi / 4, \pi-\arcsin \tilde{\kappa})\right), \\
K_{3}^{\prime}\left(\left(\vartheta_{3}^{\prime}, \varphi_{3}^{\prime}\right)=(3 \pi / 4,-\arcsin \tilde{\kappa})\right), & K_{4}^{\prime}\left(\left(\vartheta_{4}^{\prime}, \varphi_{4}^{\prime}\right)=(3 \pi / 4, \pi+\arcsin \tilde{\kappa})\right)
\end{array}
$$

In the limit $\tilde{\kappa} \rightarrow 1$, these coincide with $K_{2}$ and $K_{2}^{\prime}$ respectively. The symmetries a) and b) imply that there are only four essentially different points: $P, K_{1}, K_{2}$ and $K_{3}$. Since the singular point $P$ at the north pole lies in the interior part of the cone, it is not of physical interest.

Before discussing the remaining singular points we would like to make the following remark: For the three points $K_{i}(i=1,2,3)$ there exists a separatrix which goes from $K_{i}$ straight down to the origin $(0,0,0)$, moving on the surface of the cone. In fact, Eqs. (3.17) have admit solutions of the form $(\vartheta(\tau), \varphi(\tau)) \equiv\left(\vartheta_{i}, \varphi_{i}\right)$, where $\left(\vartheta_{i}, \varphi_{i}\right)$ denotes the angular coordinates of $K_{i}$, along which we have $\rho_{\tau} \leq 0$. This is not contradictory to the statements made in section 3.1, because the scale factor $\tilde{a}$ vanishes along these separatrices.

\subsubsection{The Singular Point $\mathrm{K}_{1}$}

In order to study the asymptotic behavior of the solutions near $K_{1}$ we first perform a shift, of the variable, defined by $\delta \rho=\rho-1, \delta \vartheta=\vartheta-\vartheta_{1}$ and $\delta \varphi=\varphi-\varphi_{1}$. In these new coordinates, 
the linearization of Eqs. (3.17) at $K_{1}$ gives

$$
\left(\begin{array}{l}
\delta \rho \\
\delta \vartheta \\
\delta \varphi
\end{array}\right)_{\tau}=\left(\begin{array}{ccc}
\lambda_{\rho} & 0 & 0 \\
0 & \lambda_{\vartheta} & 0 \\
0 & 0 & \lambda_{\varphi}
\end{array}\right)\left(\begin{array}{l}
\delta \rho \\
\delta \vartheta \\
\delta \varphi
\end{array}\right)
$$

with the strictly positive eigenvalues $\lambda_{\rho}=\sqrt{2} / 2, \lambda_{\vartheta}=2 \sqrt{2}$ and $\lambda_{\varphi}=\sqrt{2} / 2(1+\tilde{\kappa})$. Hence, the singular point $K_{1}$ is hyperbolic [8, p. 69] and the leading terms near $K_{1}$ are given by

$$
\begin{aligned}
& (\delta \rho)(\tau)=-(\delta \rho)_{o} e^{\lambda_{\rho} \tau}, \quad(\delta \vartheta)(\tau)=(\delta \vartheta)_{o} e^{\lambda_{\vartheta} \tau} \\
& (\delta \varphi)(\tau)=(\delta \varphi)_{o} e^{\lambda_{\varphi} \tau} \quad(\tau \rightarrow-\infty)
\end{aligned}
$$

with arbitrary integration constants $(\delta \rho)_{o}>0,(\delta \vartheta)_{o} \geq 0$ and $(\delta \varphi)_{o}>0$. Integration of Eq. (3.16) leads to the relation $e^{\lambda_{\rho} \tau}=\frac{\lambda_{\rho}}{(\delta \rho)_{o}} \eta$, i.e., emergence from $K_{1}$ corresponds to increasing $\eta$, starting from $\eta=0$.

For the Cartesian coordinates $(u, y, z)$ we obtain (indicating only the leading terms as $\left.\eta \rightarrow 0^{+}\right)$

$$
\begin{aligned}
u(\eta) & =(\delta \varphi)_{o}\left[\frac{\lambda_{\rho}}{(\delta \rho)_{o}}\right]^{1+\tilde{\kappa}} \eta^{\tilde{\kappa}}, \\
y(\eta) & =-\frac{1}{\eta} \\
z(\eta) & =\frac{1}{\eta} .
\end{aligned}
$$

From Eq. (3.11) it is readily seen that near $K_{1}$ the scale factor follows the law $\tilde{a}(\eta)=C \eta$, where $C>0$ is a constant, fixed by the constraint (3.10).

Hence, to the point $K_{1}$ there corresponds an initial cosmological singularity at a certain finite time which can always be chosen at $t=0$. Near $K_{1}$ we have

$$
H=\frac{1}{2 t}, \quad \dot{\phi}=-\sqrt{\frac{3}{4 G}} \frac{1}{t}, \quad \phi=-\sqrt{\frac{3}{4 G}} \ln \left(\frac{t}{t_{o}}\right),
$$

where $t_{o}>0$ is an arbitrary constant. Emergence from $K_{1}$ corresponds to increasing $t$, beginning from $t=0$. The effective equation of state near $K_{1}$ tends to $\epsilon=p$ (i.e., stiff matter).

\subsubsection{The Singular Point $\mathrm{K}_{2}$}

In order to study the asymptotic behavior of the solutions near $K_{2}$ we proceed in a similar manner. Linearization at $K_{2}$ again leads to Eq. (3.18) with the same eigenvalues $\lambda_{\rho}$ and $\lambda_{\vartheta}$, but with the changed eigenvalue $\lambda_{\varphi}=\sqrt{2} / 2(1-\tilde{\kappa})$. We suspect that in this case the behavior may depend on the value of $\tilde{\kappa}$. For $\tilde{\kappa}<1$ all eigenvalues are again strictly positive. Hence, the singular point $K_{2}$ is hyperbolic and the leading terms in the expansion around 
$K_{2}$ are given by Eq. (3.19) with arbitrary constants $(\delta \rho)_{o}>0,(\delta \vartheta)_{o} \geq 0$ and $(\delta \varphi)_{o}<$ 0 . Integration of Eq. (3.16) leads to the relation $e^{\lambda_{\rho} \tau}=\frac{\lambda_{\rho}}{(\delta)_{o}} \eta$, i.e. emergence from $K_{2}$ corresponds to increasing $\eta$, beginning from $\eta=0$.

For the Cartesian coordinates $(u, y, z)$ we obtain in leading order as $\eta \rightarrow 0^{+}$

$$
\begin{aligned}
& u(\eta)=-(\delta \varphi)_{o}\left[\frac{\lambda_{\rho}}{(\delta \rho)_{o}}\right]^{1-\tilde{\kappa}} \eta^{-\tilde{\kappa}}, \\
& y(\eta)=\frac{1}{\eta} \\
& z(\eta)=\frac{1}{\eta} .
\end{aligned}
$$

Near $K_{2}$ the scale factor follows the law $\tilde{a}(\eta)=C \eta$, where $C>0$ is a constant fixed by the constraint (3.10). Hence, if $\tilde{\kappa}<1, K_{2}$ corresponds to an initial cosmological singularity at a finite time $t=0$, say. Near $K_{2}$ we have

$$
H=\frac{1}{2 t}, \quad \dot{\phi}=\sqrt{\frac{3}{4 G}} \frac{1}{t}, \quad \phi=\sqrt{\frac{3}{4 G}} \ln \left(\frac{t}{t_{o}}\right),
$$

where $t_{o}>0$ is an arbitrary constant. Emergence from $K_{2}$ corresponds to increasing $t$, starting from $t=0$. The equation of state tends to $\epsilon=p$ (i.e., stiff matter).

If $\tilde{\kappa}>1$, the singular point $K_{2}$ is also hyperbolic, however, $\lambda_{\varphi}$ is strictly negative. The outgoing trajectories necessarily lie on the invariant plane $u=0$ (i.e: $(\delta \varphi)_{o}=0$ ) and are thus not of interest.

The case $\tilde{\kappa}=1$ is more involved because the singular point $K_{2}$ is non-hyperbolic. Terms of higher order become important. Up to third order the expansion in a sufficiently small neighborhood around $K_{2}$ is

$$
\begin{aligned}
(\delta \rho)_{\tau}= & \frac{\sqrt{2}}{2} \delta \rho+\sqrt{2} \delta \rho^{2}+\frac{3 \sqrt{2}}{2} \delta \rho \delta \vartheta+\frac{\sqrt{2}}{2} \delta \rho^{3} \\
& -\frac{9 \sqrt{2}}{4} \delta \rho \delta \vartheta^{2}+2 \sqrt{2} \delta \rho^{2} \delta \vartheta-\frac{\sqrt{2}}{2} \delta \rho \delta \varphi^{2} \\
(\delta \vartheta)_{\tau}= & 2 \sqrt{2} \delta \vartheta+2 \sqrt{2} \delta \vartheta^{2}+2 \sqrt{2} \delta \rho \delta \vartheta \\
& -\frac{7 \sqrt{2}}{3} \delta \vartheta^{3}+2 \sqrt{2} \delta \rho \delta \vartheta^{2}-\sqrt{2} \delta \vartheta \delta \varphi^{2} \\
(\delta \varphi)_{\tau}= & -\sqrt{2} \delta \vartheta \delta \varphi-\frac{\sqrt{2}}{4} \delta \varphi^{3}-\sqrt{2} \delta \rho \delta \vartheta \delta \varphi .
\end{aligned}
$$

We can now use the Poincaré-Dulac theorem [9, p. 191] to classify the nonlinear terms in Eq. (3.24) into resonant and non-resonant ones. For the non-resonant terms of order $n$ there is a polynomial change of coordinates of degree $n$ so that they are transformed into terms of at least order $n+1$. This is not the case for the resonant terms. The polynomial 
change of coordinates is found by solving the so-called homological equation. Performing the polynomial change of coordinates on Eq. (3.24), the non-resonant terms of degree 2 and 3 become of higher order and are thus neglected. Retaining only terms up to third order, we obtain the set of equations

$$
\begin{aligned}
\frac{d v_{\rho}}{d \tau} & =\frac{\sqrt{2}}{2} v_{\rho}-\frac{\sqrt{2}}{2} v_{\rho} v_{\varphi}^{2} \\
\frac{d v_{\vartheta}}{d \tau} & =2 \sqrt{2} v_{\vartheta}-\sqrt{2} v_{\vartheta} v_{\varphi}^{2} \\
\frac{d v_{\varphi}}{d \tau} & =-\frac{\sqrt{2}}{4} v_{\varphi}^{3}
\end{aligned}
$$

where $(\delta \rho, \delta \vartheta, \delta \varphi)=\left(v_{\rho}, v_{\vartheta}, v_{\varphi}\right)+$ polynomes of degree 2 or higher in $\left(v_{\rho}, v_{\vartheta}, v_{\varphi}\right)$. The minus sign in the last equation implies that for $\tilde{\kappa}=1$ there are no trajectories which emanate from $K_{2}$ into the exterior of the cone. Indeed, Eqs. (3.25) can be solved analytically. We obtain

$$
\begin{aligned}
v_{\varphi}(\tau) & = \pm \frac{1}{\sqrt{\left(v_{\varphi o}\right)^{-2}+\frac{\sqrt{2} \tau}{2}}} \quad \tau \in\left(-\sqrt{2}\left(v_{\varphi o}\right)^{-2}, \infty\right), \\
v_{\rho}(\tau) & =\frac{v_{\rho o}}{\left(v_{\varphi o}\right)^{2}} v_{\varphi}^{2}(\tau) e^{\frac{\sqrt{2}}{2} \tau}, \\
v_{\vartheta}(\tau) & =\frac{v_{\vartheta o}}{\left(v_{\varphi o}\right)^{4}} v_{\varphi}^{4}(\tau) e^{2 \sqrt{2} \tau},
\end{aligned}
$$

where $v_{\rho o}, v_{\vartheta o}$ and $v_{\varphi_{o}} \neq 0$ are arbitrary constants. The $+(-)$ sign corresponds to the case $v_{\varphi o}>0\left(v_{\varphi o}<0\right)$. Hence, for non-vanishing constants $v_{\rho o}$ and $v_{\vartheta o}$, we have

$$
v_{\varphi}(\tau) \rightarrow 0, \quad\left|v_{\rho}(\tau)\right| \rightarrow \infty, \quad\left|v_{\vartheta}(\tau)\right| \rightarrow \infty \quad(\tau \rightarrow \infty)
$$

\subsubsection{The Singular Point $\mathrm{K}_{3}$}

The singular point $K_{3}$ only appears for $\tilde{\kappa}<1$. Linearization at $K_{3}$ leads to

$$
\left(\begin{array}{l}
\delta \rho \\
\delta \vartheta \\
\delta \varphi
\end{array}\right)_{\tau}=\left(\begin{array}{ccc}
\mu_{\rho} & 0 & 0 \\
0 & \mu_{\vartheta} & 0 \\
0 & \sqrt{2} \tilde{\kappa} \sqrt{1-\tilde{\kappa}^{2}} & \mu_{\varphi}
\end{array}\right)\left(\begin{array}{l}
\delta \rho \\
\delta \vartheta \\
\delta \varphi
\end{array}\right)
$$

with the eigenvalues $\mu_{\rho}=\sqrt{2} / 2 \tilde{\kappa}^{2}, \mu_{\vartheta}=\sqrt{2}\left(1+\tilde{\kappa}^{2}\right)$ and $\mu_{\varphi}=-\sqrt{2} / 2\left(1-\tilde{\kappa}^{2}\right)$. The point $K_{3}$ is thus hyperbolic and the leading terms near it are given by

$$
\begin{aligned}
& (\delta \rho)(\tau)=-(\delta \rho)_{o} e^{\mu_{\rho} \tau}, \quad(\delta \vartheta)(\tau)=(\delta \vartheta)_{o} e^{\mu_{\vartheta} \tau}, \\
& (\delta \varphi)(\tau)=\frac{\sqrt{2} \tilde{\kappa} \sqrt{1-\tilde{\kappa}^{2}}}{\mu_{\vartheta}-\mu_{\varphi}}(\delta \vartheta)_{o} e^{\mu_{\vartheta} \tau} \quad(\tau \rightarrow-\infty),
\end{aligned}
$$

with arbitrary constants $(\delta \rho)_{o}>0$ and $(\delta \vartheta)_{o}>0$. Note that the general solution of (3.26) would be given by

$$
(\delta \varphi)(\tau)=\frac{\sqrt{2} \tilde{\kappa} \sqrt{1-\tilde{\kappa}^{2}}}{\mu_{\vartheta}-\mu_{\varphi}}(\delta \vartheta)_{o} e^{\mu_{\vartheta} \tau}+(\delta \varphi)_{o} e^{\mu_{\varphi} \tau}
$$


However, since $\mu_{\varphi}$ is negative the constant $(\delta \varphi)_{o}$ must vanish.

Integration of Eq. (3.16) leads to the relation $e^{\mu_{\rho} \tau}=\frac{\mu_{\rho}}{(\delta \rho)_{o}} \eta$, i.e., emergence from $K_{3}$ corresponds to increasing $\eta$, starting from $\eta=0$. In terms of the Cartesian coordinates $(u, y, z)$ we obtain

$$
\begin{aligned}
u(\eta)= & \tilde{\kappa}^{-2} \sqrt{1-\tilde{\kappa}^{2}} \frac{1}{\eta}-\sqrt{\left(1-\tilde{\kappa}^{2}\right) / 2} \\
& +\tilde{\kappa}^{-2} \sqrt{1-\tilde{\kappa}^{2}} \frac{3-\tilde{\kappa}^{2}}{3+\tilde{\kappa}^{2}}(\delta \vartheta)_{o}\left[\frac{\mu_{\rho}}{(\delta \rho)_{o}}\right]^{\frac{\mu_{\vartheta}}{\mu_{\rho}}} \eta^{\frac{\mu_{\vartheta}}{\mu_{\rho}}-1}, \\
y(\eta)= & \tilde{\kappa}^{-1} \frac{1}{\eta}-\frac{\sqrt{2}}{2} \tilde{\kappa}+\tilde{\kappa}^{-1} \frac{5-\tilde{\kappa}^{2}}{3+\tilde{\kappa}^{2}}(\delta \vartheta)_{o}\left[\frac{\mu_{\rho}}{(\delta \rho)_{o}}\right]^{\frac{\mu_{\vartheta}}{\mu_{\rho}}} \eta^{\frac{\mu_{\vartheta}}{\mu_{\rho}}-1}, \\
z(\eta)= & \tilde{\kappa}^{-2} \frac{1}{\eta}-\frac{\sqrt{2}}{2}-\tilde{\kappa}^{-2}(\delta \vartheta)_{o}\left[\frac{\mu_{\rho}}{(\delta \rho)_{o}}\right]^{\frac{\mu_{\vartheta}}{\mu_{\rho}}} \eta^{\frac{\mu_{\vartheta}}{\mu_{\rho}}-1},
\end{aligned}
$$

with $\frac{\mu_{\vartheta}}{\mu_{\rho}}=2\left(1+\tilde{\kappa}^{-2}\right)>4$. From Eq. (3.11) it is readily seen that near $K_{3}$ the scale factor behaves like $\tilde{a}(\eta)=C \eta^{1 / \tilde{\kappa}^{2}}$, where $C>0$ is a constant, fixed by the constraint (3.10).

The point $K_{3}$ therefore corresponds to an initial cosmological singularity at a finite time $t=0$, say. Near $K_{3}$ we have

$$
H=\left(1+\tilde{\kappa}^{2}\right)^{-1} \frac{1}{t}, \quad \dot{\phi}=\frac{\tilde{\kappa}}{1+\tilde{\kappa}^{2}} \sqrt{\frac{3}{G}} \frac{1}{t}, \quad \phi=\frac{\tilde{\kappa}}{1+\tilde{\kappa}^{2}} \sqrt{\frac{3}{G}} \ln \left(\frac{t}{t_{o}}\right),
$$

where $t_{o}>0$ is an arbitrary constant. Emergence from $K_{3}$ corresponds to increasing $t$, starting from $t=0$.

\section{Numerical Results}

The asymptotic behavior of the solutions near the singular points provides one with the initial conditions for solving the differential equations numerically. We will recover the repelling $\left(K_{1}, K_{2}, K_{3}\right)$ and attracting $\left(K_{1}^{\prime}, K_{2}^{\prime}, K_{3}^{\prime}\right)$ characteristics of the singular points, as well as their dependence on the value of $\tilde{\kappa}$. Special emphasis is given to the value $\tilde{\kappa}=\sqrt{3}$ which is predicted by a model obtained from superstring theory [2, 10].

\subsection{Emergence from $\mathrm{K}_{1}$}

The asymptotic behavior (3.20) of the solutions near $K_{1}$ provides proper initial conditions at a given time $\eta=\eta_{o}$. The constraint is taken into account by fixing the constant $C$ at the time $\eta_{o}$. We can now produce plots for different values of $\tilde{\kappa}$ and $k:=(\delta \varphi)_{o}\left[\frac{\lambda_{\rho}}{(\delta \rho)_{o}}\right]^{1+\tilde{\kappa}}$. 
$\tilde{\kappa}=1 / 2$ : Fig. 4 shows the behavior of the scale factor $\tilde{a}$ for different values of the constant $k$. The phase portrait of the dilaton field is given in Fig. 5 . Since $\tilde{\kappa}<1$, each singular point $K_{1}^{\prime}, K_{2}^{\prime}$ or $K_{3}^{\prime}$ is attractive. From Fig. 6 it can be seen that the plots actually correspond to
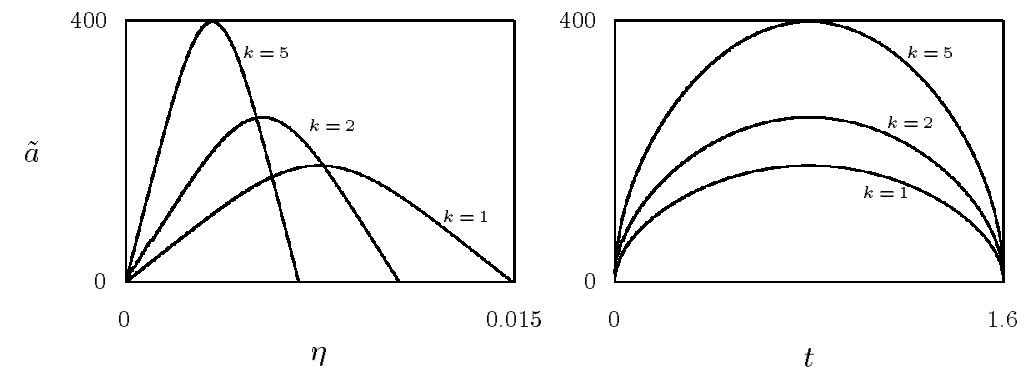

Figure 4: The scale factor with respect to the time $\eta$ and the conformal time $t$ respectively.

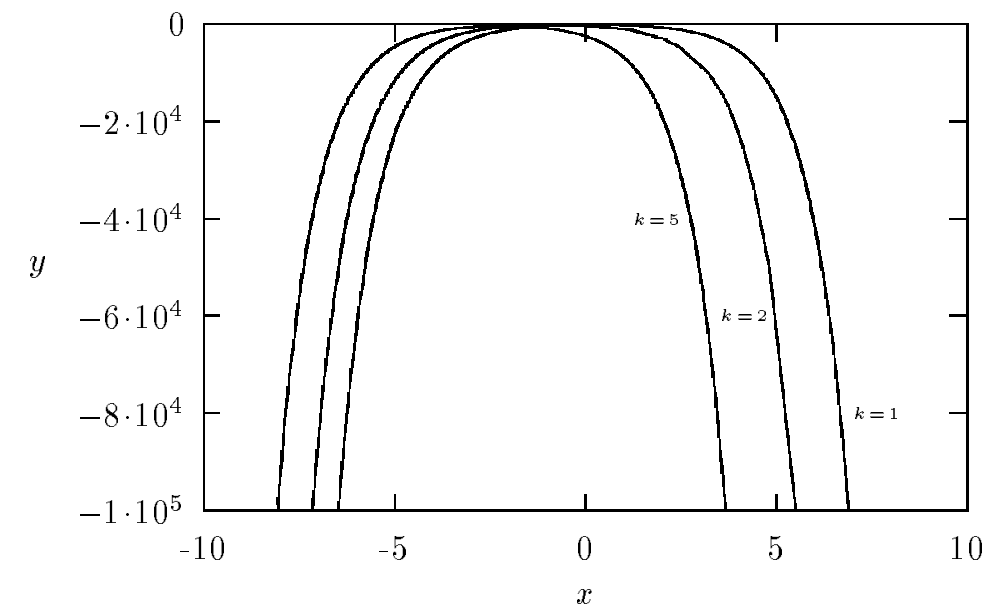

Figure 5: Phase portrait of the dilaton field.
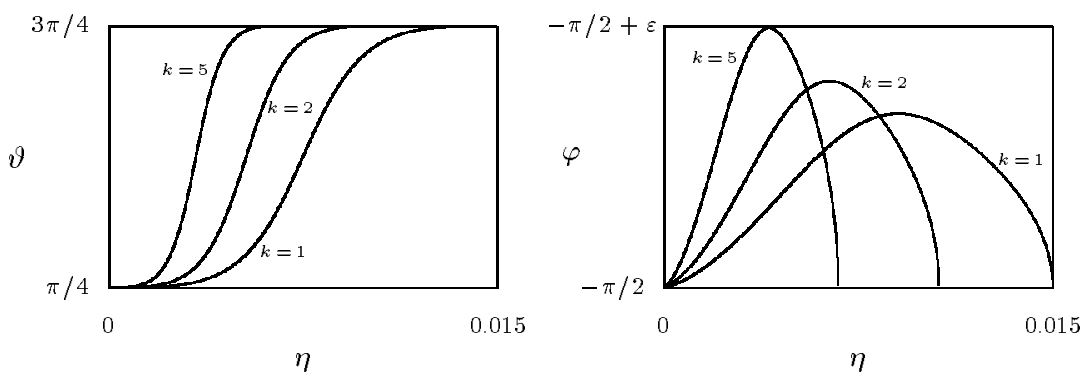

Figure 6: The behavior of $\vartheta$ and $\varphi$ with respect to $\eta$. The value of $\varepsilon$ is $\varepsilon=8 \cdot 10^{-4}$. The trajectories are therefore close to the plane $u=0$.

trajectories which end at $K_{2}^{\prime}$. We have also computed numerically $\vartheta$ and $\varphi$ as a function of time for all subsequent cases. This enable us to identify the points at which the trajectories 
end. These plots can be found in the Diploma thesis of one of us [12].

$\tilde{\kappa}=\sqrt{3}:$ Fig. 7 shows the behavior of the scale factor $\tilde{a}$ for different values of the constant $k$. The phase portrait of the dilaton field is given in Fig. 8. Since for $\tilde{\kappa} \geq 1$ only $K_{1}^{\prime}$ is an attractive point, we expect that the plots correspond to trajectories which end at $K_{1}^{\prime}$. This is confirmed by our numerical computation.
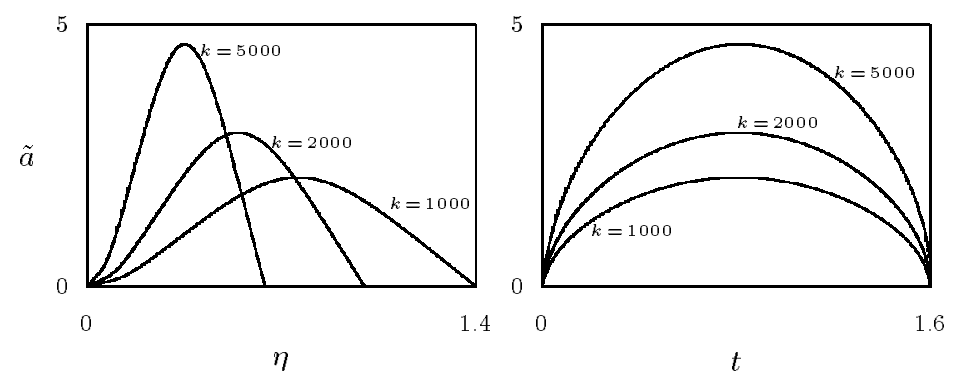

Figure 7: The scale factor with respect to the time $\eta$ and the conformal time $t$ respectively.

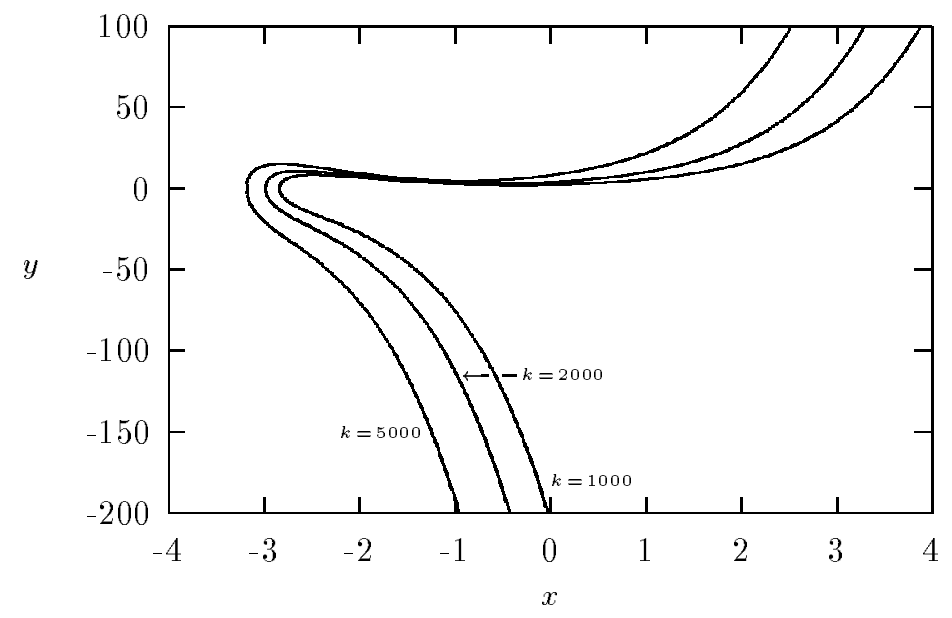

Figure 8: Phase portrait of the dilaton field.

\subsection{Emergence from $\mathrm{K}_{2}$}

We proceed in a similar manner. Emergence from $K_{2}$ only appears if $\tilde{\kappa}<1$. The constant $k$ is defined by $k:=-(\delta \varphi)_{o}\left[\frac{\lambda_{\rho}}{(\delta \rho)_{o}}\right]^{1-\tilde{\kappa}}$ (see Eq. (3.22)).

$\tilde{\kappa}=1 / 2$ : Fig. 9 shows the behavior of the scale factor $\tilde{a}$ for different values of the constant $k$. The phase portrait of the dilaton field is given in Fig. 10. The plots correspond to trajectories which end at the singular point $K_{1}^{\prime}$. 

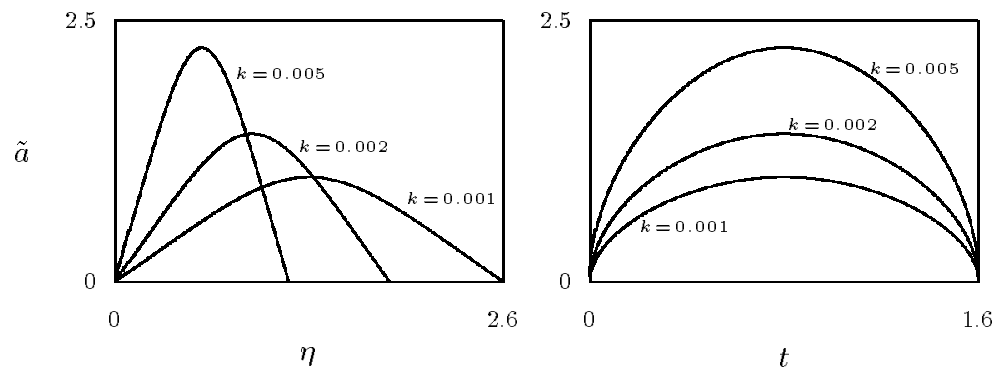

Figure 9: The scale factor with respect to the time $\eta$ and the conformal time $t$ respectively.

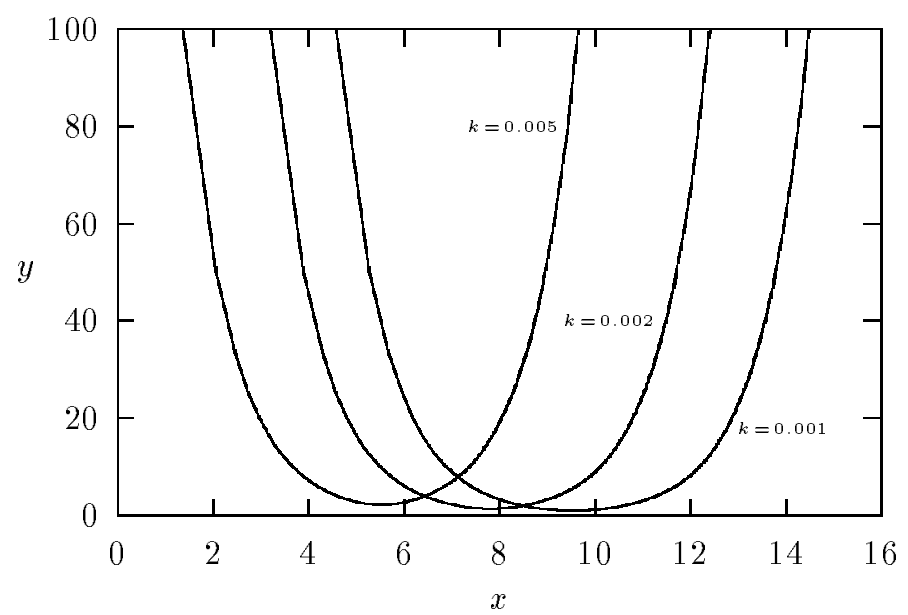

Figure 10: Phase portrait of the dilaton field.

\subsection{Emergence from $\mathrm{K}_{3}$}

The singular point $K_{3}$ only exists if $\tilde{\kappa}<1$. We define the constant $k$ by $k:=(\delta \vartheta)_{o}\left[\frac{\mu_{\rho}}{\left(\delta \rho_{o}\right.}\right]^{\frac{\mu_{\vartheta}}{\mu_{\rho}}}$ (seeEq. (3.28)).

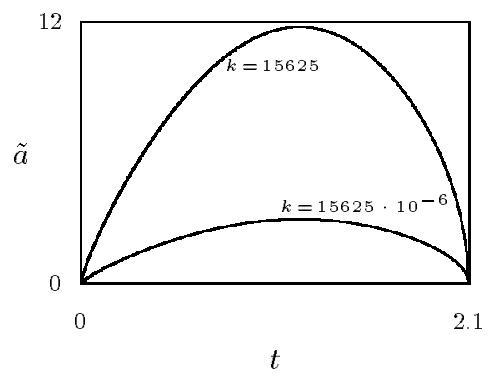

Figure 11: The scale factor with respect to the conformal time $t$. 
$\tilde{\kappa}=1 / 2$ : Fig. 11 shows the behavior of the scale factor $\tilde{a}$ for different values of the constant $k$. The phase portrait of the dilaton field is given in Fig. 12. The plots correspond to trajectories which end at $K_{1}^{\prime}$.

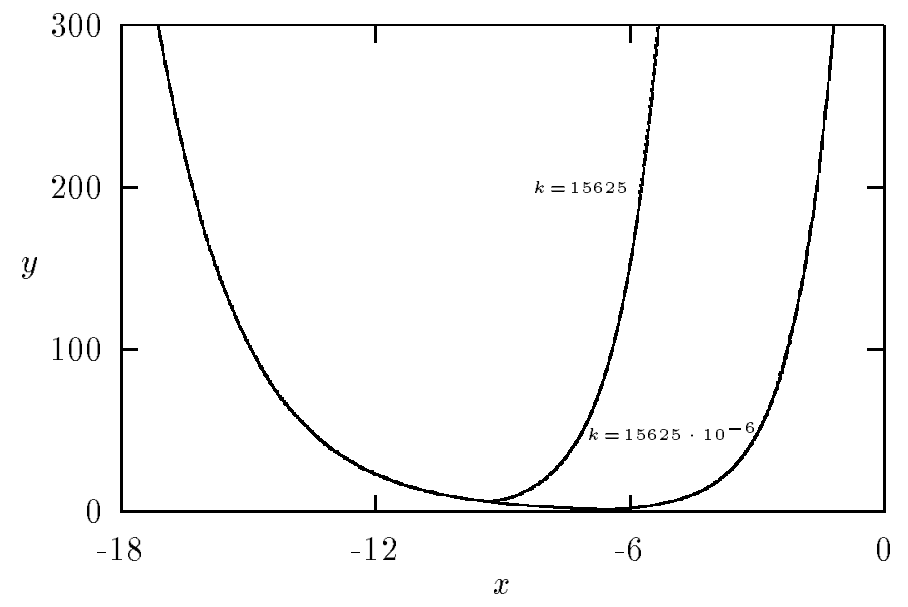

Figure 12: Phase portrait of the dilaton field.

\section{Concluding Remarks}

For the special case of a static gauge field and $V=\Lambda=0$ we have performed a complete analysis of the coupled EYMD equations for a Friedmann-Lemaitre universe with constant curvature $k=1$ (closed model). For this situation, we did not recover any inflationary stages. It might therefore be interesting to extend our discussion to the more general case of a dynamical gauge field, perhaps with a non-vanishing cosmological constant $\Lambda$ and/or a dilaton potential $V$. This is, however, a considerably more involved task, because we encounter then six coupled first-order nonlinear differential equations.

The analysis of the coupled EYMD equations for a Friedmann-Lemaitre universe with constant curvature $k=0$ (flat model) and $k=-1$ (open model) are also of interest, since related considerations [7, 11] show that inflationary stages occur for trajectories which come close enough to one of the separatrices found for $k=0$.

\section{Acknowledgments}

We are grateful to Othmar Brodbeck, George Lavrelashvili and Mikhail Volkov for very useful and clarifying discussions, and to Marcus Heusler for careful reading of the manuscript. 


\section{References}

[1] A.Linde, Particle Physics and Inflationary Cosmology (Harwood Academic Publishers, Chur).

[2] M.B.Green, J.H.Schwarz and E.Witten, Superstring Theory, Vol. 2 (Cambridge U.P., Cambridge, 1988).

[3] R.B.Abbot, B.Bednarz and S.D.Ellis, Phys. Rev. D 33, 2147 (1986).

[4] G.Veneziano, CERN-TH/95-254, September 1995.

[5] M.C.Bento and O.Bertolami, CERN-TH/95-36, DFTT 20/95, April 1995

[6] Y. Hosotani, Phys. Lett. B 147 (1984) 44.

M. Henneaux, Journ. Math. Phys. 23 (1982) 830.

G.W.Gibbons and Alan R.Steif, Physics Letters, B 320, 245-252 (1994).

[7] D.Scialom and P.Jetzer, Phys. Rev. D 51, 5698-5706 (1995).

[8] D.K.Arrowsmith and C.M.Place, An Introduction to Dynamical Systems (Cambridge, Cambridge University Press, 1990).

[9] V.I.Arnold, Geometrical Methods in the Theory of Ordinary Differential Equations (Springer-Verlag, New York, 1983).

[10] G.Lavrelashvili and D.Maison, Nucl. Phys. B 410, 407-422 (1993).

[11] V.A.Belinsky, L.P.Grishchuk, Ya.B.Zeldovich and I.M.Khalatnikov, Sov. Phys. JETP 62, 195 (1985).

[12] R. Eggenschwiler, Diploma Thesis, Zürich University. 